\title{
Reliability Evaluation of a Distribution System with wind Turbine Generators Based on the Switch-section Partitioning Method
}

\author{
Hongbin $\mathrm{Wu}^{\dagger}$, Jinjin Guo* and Ming Ding*
}

\begin{abstract}
Considering the randomness and uncertainty of wind power, a reliability model of WTGs is established based on the combination of the Weibull distribution and the Markov chain. To analyze the failure mode quickly, we use the switch-section partitioning method. After defining the first-level load zone node, we can obtain the supply power sets of the first-level load zone nodes with each WTG. Based on the supply sets, we propose the dynamic division strategy of island operation. By adopting the fault analysis method with the attributes defined in the switch-section, we evaluate the reliability of the distribution network with WTGs using a sequential Monte Carlo simulation method. Finally, using the IEEE RBTS Bus6 test system, we demonstrate the efficacy of the proposed model and method by comparing different schemes to access the WTGs.
\end{abstract}

Keywords: Distribution network, Wind Turbine Generator (WTG); Reliability evaluation, Switchsection partitioning method, Sequential Monte Carlo simulation

\section{Introduction}

With the development of wind power generation technology, an increasing number of wind turbine generators (WTG) are accessing the distribution network. There are large differences in the structure and operation modes of traditional distribution networks, depending on whether they include WTGs, so the traditional distribution network reliability evaluation method [1-2] is not suitable for WTGs. Instead, we must consider the characteristic randomness and uncertainty of wind energy in the modeling process. Meanwhile, the inclusion of WTGs will likely cause the island operation mode to appear in the failure analysis process. Therefore, it is necessary to study a suitable power supply reliability evaluation method for distribution networks with WTGs.

The reliability evaluation of the distribution network mainly includes two analysis methods: analytic and simulation. The analytical method [3-5] calculates the system reliability index using the mathematical model of reliability and analyzes the expected failure, whereas the simulation method [6-8] chooses the system state using the method of sampling status and uses a statistical method to calculate the reliability index. For a complex distribution network, it is difficult to obtain an accurate mathematical model using the analytical method, whereas the simulation method is not subject to limitations of network scale. Thus, the paper adopts the simulation method to study the reliability evaluation of distribution networks with WTGs.

$\dagger$ Corresponding Author: School of Electrical Engineering and Automation, Hefei University of Technology, Hefei 230009, China. (hfwuhongbin@163.com)

* School of Electrical Engineering and Automation, Hefei University of Technology, Hefei 230009, China.

Received: May 9, 2014 ; Accepted: December 1, 2015
Because of the randomness and uncertainty of wind energy, it is necessary to establish a model of WTGs that is suitable for reliability evaluation. In [9], a wind turbine reliability model was established based on the analytical frequency and duration approach, taking into consideration the randomness of wind speed; and given the probability and duration of the wind turbine output power. However, this model did not consider the effect of wind turbine faults on its active power output, and the model was inconsistent with the actual operation. A wind turbine reliability model was established that incorporated the randomness of wind power in [10-11]. However, this model did not include the derated state of the turbine, which would introduce errors in the prediction. In addition, it could only provide the probability of wind power output, and not its duration, so it was not amenable to sequential Monte Carlo simulation.

After WTGs are connected to the power distribution network, the operation mode and structure of the distribution network changes. The analysis of traditional failure mode effects has not incorporated the island operation mode, so it cannot properly consider wind power supply in some failure cases. As a result, it is necessary to study the island dynamic division strategy and establish a kind of fault mode analysis method for distribution networks that includes the wind power effect. In [12-13], the analytical method was used for a reliability evaluation of the distribution system in different models, considering the randomness of wind power. However, this analysis presupposed that an island could only be formed when no WTG failure occurred and when the generating capacity of the WTG was greater than or equal to the load demand. Wind power could not be fully used, and the power supply path for the first-level load is not established. In [14-15], an efficient and practical reliability evaluation algorithm was presented 
for distribution networks using a section technique and accounting for the construction features of the distribution networks. However, the zone partition method in this paper cannot address the effect of wind power on the power supply area of the distribution network because of the random variations in the output of wind power. The failure effect table of regional components was obtained using a simplified network in [16], which facilitated a reliability evaluation of the distribution network containing DG based on the sequential Monte Carlo method. However, this method simply illustrates the development of the fault effect table, and there is no detailed introduction to the process of failure mode analysis.

Taking into account the above problems, in this paper, a reliability evaluation of distribution systems with WTGs is presented. A reliability model of WTGs is proposed based on a combination of the Weibull distribution and the Markov chain, which is suitable for sequential Monte Carlo simulation. To quickly analyze the failure mode, we use the concept of switch-section and propose the switch-section search method for a distribution network that includes only one layer of child feeders. After defining the first-level load zone node, we obtain the supply power sets of the firstlevel load zone nodes with each WTG. Based on the supply sets, we propose the dynamic division strategy of island operation. By adopting a fault analysis method based on the attributes of the switch-section, we evaluate the reliability of the distribution network with WTGs using the sequential Monte Carlo simulation method. Finally, with the IEEE RBTS Bus6 test system, the proposed model and method are validated, and we discuss various effects on the reliability indices of distribution networks with WTGs.

\section{Reliability Evaluation Model of WTGs}

\subsection{Wind energy transformation model based on the Weibull distribution}

The wind speed at a wind farm is random, and a large number of experimental data analysis results show that the wind speed obeys a two-parameter Weibull distribution. Using the wind speed probability statistics model, i.e., the Weibull distribution, it is possible to transfer the randomness of the wind speed to the wind power output. Its probability density function is:

$$
f(v)=\frac{k}{c}\left(\frac{v}{c}\right)^{k-1} \exp \left[-\left(\frac{v}{c}\right)^{k}\right]
$$

The probability distribution function can be obtained from formula (1):

$$
F(v)=P(V \leq v)=1-\exp \left[-\left(\frac{v}{c}\right)^{k}\right]
$$

where $c$ and $k$ are the scale and shape parameters, respectively.

According to the counter transformation method, the incident wind speed is described as:

$$
v_{i}=-c \ln \left(\alpha_{i}\right)^{\frac{1}{k}}
$$

where $\alpha_{i}$ is a evenly distributed variable in the interval $[0,1]$.

The model uses a time step of one hour, and generates a random number $\alpha_{i}$ in the interval $[0,1]$ in each hour interval. The wind energy transformation model $P_{w}[t]$ can then be obtained as follows:

$$
P_{w}[t]= \begin{cases}0 & \left(\alpha_{i}<e^{-\left(\frac{v_{c o}}{c}\right)^{k}}\right) \cup\left(\alpha_{i}>e^{-\left(\frac{v_{c i}}{c}\right)^{k}}\right) \\ P_{n} \frac{c\left(-\ln \left(\alpha_{i}\right)\right)^{\frac{1}{k}}-v_{c i}}{v_{n}-v_{c i}} & e^{-\left(\frac{v_{n}}{c}\right)^{k}} \leq \alpha_{i}<e^{-\left(\frac{v_{c i}}{c}\right)^{k}} \\ P_{n} & e^{-\left(\frac{v_{c o}}{c}\right)^{k}} \leq \alpha_{i}<e^{-\left(\frac{v_{n}}{c}\right)^{k}}\end{cases}
$$

where $v_{n}$ is the rated wind speed, $v_{c i}$ is the cut-in wind speed, $v_{c o}$ is the removal speed of wind, $P_{n}$ is the rated power, and $t$ is the simulation time.

\subsection{Output power coefficient of WTGs based on the Markov chain}

Markov chains [17] are typically used for random process modeling. For the WTG, the chain has three states: Stop, Derated and Available. Fig. 1 shows the three-state WTG model.

In Fig. 1, $\lambda$ represents the transfer rate among the states, and the subscript indicates the status of the transfer direction. For the three-state model of the WTG, it is possible to obtain the state transition matrix by adopting Markov chain methods:

$$
Z_{n}=\left[\begin{array}{ccc}
1-\lambda_{A D}-\lambda_{A S} & \lambda_{A D} & \lambda_{A S} \\
\lambda_{D A} & 1-\lambda_{D A}-\lambda_{D S} & \lambda_{D S} \\
\lambda_{S A} & \lambda_{S D} & 1-\lambda_{S D}-\lambda_{S A}
\end{array}\right]
$$

According to the Markov approximation principle,

$$
P_{A}+P_{D}+P_{S}=1
$$

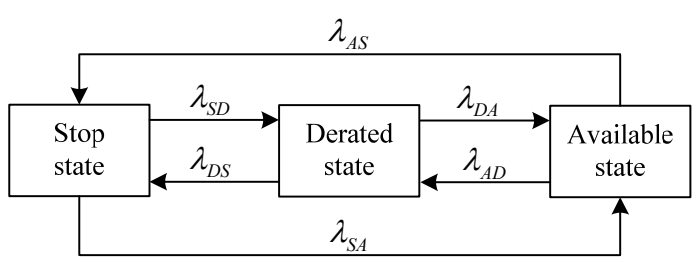

Fig. 1. Three-state WTG model 
The probability of each state $\left(P_{A}, P_{D}, P_{S}\right)$ can be calculated from formulas (5) and (6). With the sampling method of system state transition, we can sum the possible state probabilities of WTGs over the $[0,1]$ interval. We randomly generate a number $\beta_{1}$ that satisfies the requirement of uniform distribution on $[0,1]$; then, based on the law of large numbers, we can obtain WTG state, $S$, using formula (7).

$$
S= \begin{cases}\text { Stop state } & 0 \leq \beta_{1}<P_{S} \\ \text { Derate state } & P_{S} \leq \beta_{1}<P_{S}+P_{D} \\ \text { Available state } & P_{S}+P_{D} \leq \beta_{1} \leq 1\end{cases}
$$

Suppose that each state has a corresponding output power coefficient $\gamma$.

$$
\gamma= \begin{cases}0 & S=\text { Stop state } \\ 0.6 & S=\text { Derated state } \\ 1 & S=\text { Available state }\end{cases}
$$

If the state of the WTGs is $S$, the state duration $T_{s}$ can be calculated using formula (9):

$$
T_{s}=-\left(\frac{1}{\sum_{j=1}^{M_{d}} \lambda_{s j}}\right) \ln \left(\beta_{2}\right)
$$

where $M_{d}$ is the transfer number that leaves state $S, \beta_{2}$ is a random number that satisfies the $[0,1]$ uniform distribution requirement.

To obtain the output power coefficient sequence of WTGs, we define the output power coefficient array State $[t]$. The flow chart describing the process for calculating State $[t]$ is shown in Fig. 2.

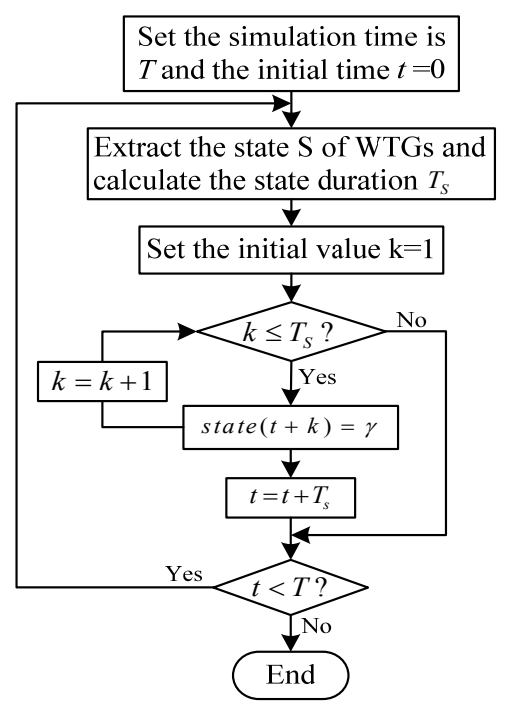

Fig. 2. Flow chart of calculating the output power coefficient of WTGs

\subsection{Reliability model of WTGs}

The reliability model of WTGs can be described as follows:

$$
P(t)=P_{w}[t] * \text { State }[t]
$$

where $P(t)$ is the output power reliability model, $P_{w}[t]$ is the wind energy transformation model from subsection 2.1 , State $[t]$ is the output power coefficient array from subsection 2.2, and $t$ is the time from 1 8760 hours.

By adopting the combination of the Weibull distribution and the Markov chain, the resultant model can be used to evaluate the reliability of the distribution network with WTGs based on sequential Monte Carlo simulation, and this model overcomes the limitations inherent to traditional wind turbine reliability models.

\section{Section Partitioning Method Based on the Switch Characteristics}

\subsection{Section partitioning method based on the switch characteristics}

The switch-section partitioning method divides the system into multiple sections based on the boundaries of the switches [18]. In a complex distribution network, it is possible to significantly reduce the computational burden of the failure analysis by using the switch-section as a unit for the failure analysis.

In a distribution network with only one layer of child feeders, and using the circuit breaker as the border, the system section types can be divided into the main feeder section (labeled F-section) and child feeder section (labeled $C_{i}$-section, where $i$ is the number of the child feeder section). Using this paradigm, we can further divide within each section, and we regard any non-isolated switching and collection of interconnected elements in the section as the load-range section, labeled $A_{j}$-section, where $j$ is the number of the load-range section. If the WTGs are contained in a certain section, we redefine the section type as $W_{k}$-section, where $k$ is the number of the section.

\subsection{Search method based on the switch characteristics}

Fig. 3 depicts the search method of the switch-section for a distribution network with only one layer of child feeders.

Taking the switch-section search method for a distribution network with only one layer of child feeders as a recurrence relation, we can conveniently describe the switch-section of a complex distribution network with $K$ $(K>1)$ layers of child feeders. 


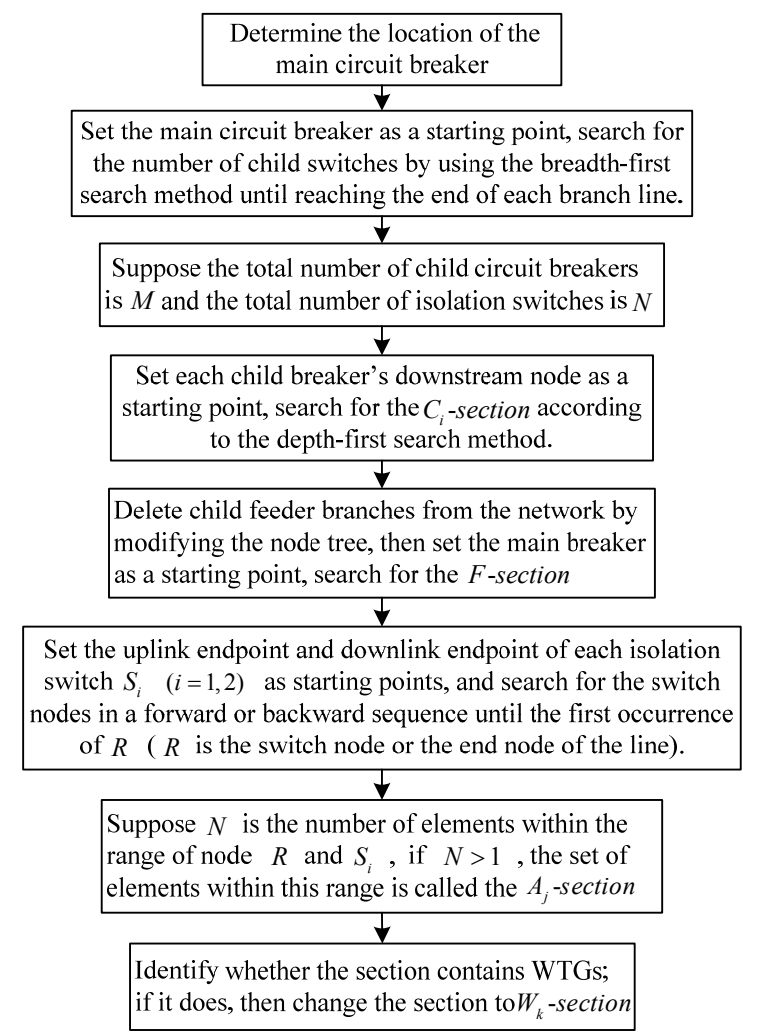

Fig. 3. Flow chart of the switch-section search method

In the search process, we have preserved the regional attributes of the switch-sections in the array, which will be helpful in reducing the failure mode analysis. A detailed description is provided in section 5 .

\section{Dynamic Division Strategy using Islands}

\subsection{First-level load zone node}

In the distribution network, the first-level load refers to the most important power load; a power failure or sudden power failure will cause great losses to the user of the firstlevel load. The distribution network node that connects to the first-level load is called the first-level load node.

A distribution network with WTGs can easily be divided into various switch-section types using the switch-section search method described in section 3. For the $W_{k}$-section, with regard to the importance of the first-level load in the distribution network and the limited ability of the wind power supply, it is necessary to supply power to the firstlevel load primarily in the $W_{k}$-section under the island mode. Therefore, in this section, we propose the concept of the first-level load zone node. We then mark out the first level load zones in each $W_{k}$-section. Using this technique, we dynamically divide the island in the $W_{k}$-section .

Definition: Set each first-level load as the starting point, and search for nearby WTGs. Determine whether the path between the power node and load node is intact. If the pathway is intact, then the first-level load can be supplied by the WTGs, and all loads between the first-level load node and the WTGs node must be supplied. Set these loads as supplementary load. The collection of the first-level load node and supplementary load nodes is called the first-level load zone node, which is marked as $T_{i-j}$, where $i$ is the number of the first-level load zone node, $j$ is the number of the first-level load node.

An example of the first-level load zone node is shown in Fig. 4.

In Fig. 4(a), LP6, LP10 and LP12 are the first-level load. In Fig. 4(b), 1, 2 and 7 are the load node units, which consist of the load, load access points and connecting lines. According to the first-level load zone nodes, we can simplify the structure of the distribution network by using $T_{1-6}=[4,5,6], T_{2-10}=[8,9,10]$, and $T_{3-12}=[11,12]$. $T_{1-6}$ is supplied by WTG1, $T_{3-12}$ is supplied by WTG2, $T_{2-10}$ can be supplied by both WTG1 and WTG2. Then, we can obtain the power supply node sets based on the firstlevel load zone nodes, where WTG1 is for $T_{1-6}$ and $T_{2-10}$, WTG2 is for $T_{2-10}$ and $T_{3-12}$. Finally, considering the realtime output power of WTG1 and WTG2, we can mark off

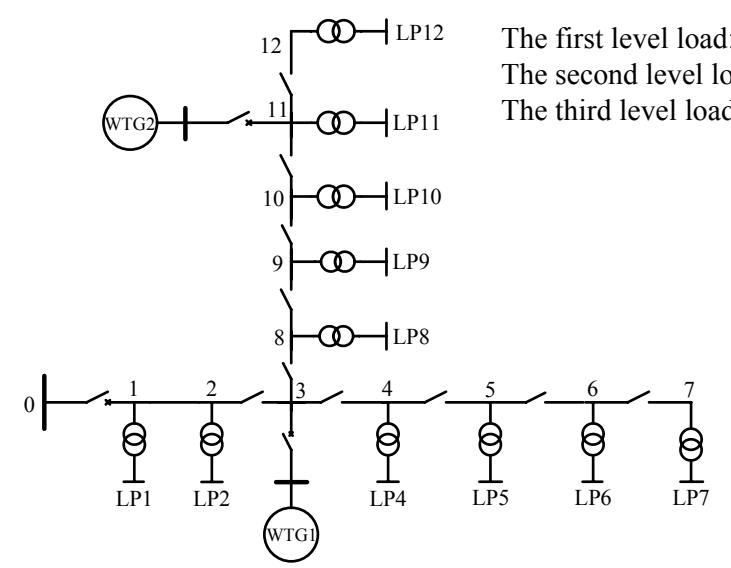

(a) Original system
LP6, LP10, LP12

LP1, LP2, LP7, LP8, LP11

LP4, LP5, LP9:

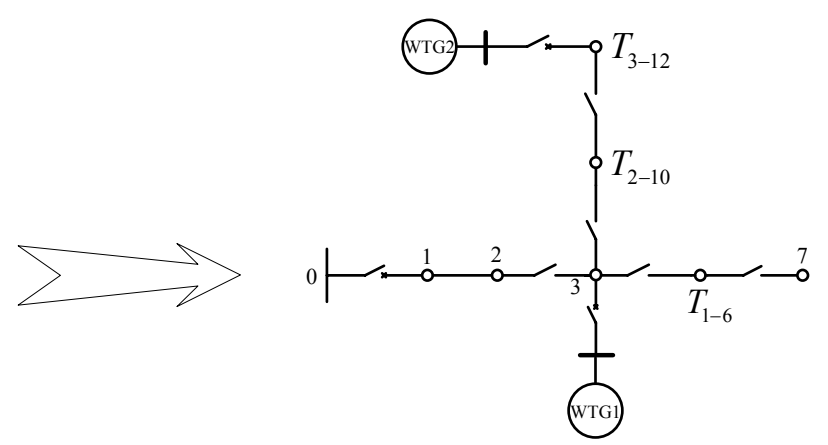

(b) First-level load regional nodes

Fig. 4. Example of the first level load zone node 
the island range online based on the island dynamic division strategy.

\subsection{Dynamic division strategy for island operation mode}

The so-called dynamic division strategy refers to cases when the island range undergoes dynamic change. This division is subject to constraints on the randomness of the output power of WTGs and the time-varying nature of the load. This division strategy for distribution networks requires a relatively high level of automation.

The detailed dynamic division constraints of an island are as follows:

$$
\left\{\begin{array}{l}
\sum_{i \in D} P_{L i}<\sum_{j \in D} P_{G j} \quad \text { Region } D \text { is connected } \\
\max P=\sum_{i \in D} w_{i} P_{L i} \\
\min M_{i}
\end{array}\right.
$$

where $D$ is the region formed by all nodes in the island, $L_{i}$ is the first-level load zone node or the set of its internal nodes, $P_{L i}$ is the active power of the load, $P_{G j}$ is the output power of WTGs, $w_{i}$ is the weighted coefficient of load $i, P$ is the weighted sum of the power load in the island, and $M_{i}$ is the total quantity of the removed load.

A flow chart of the dynamic island division procedures

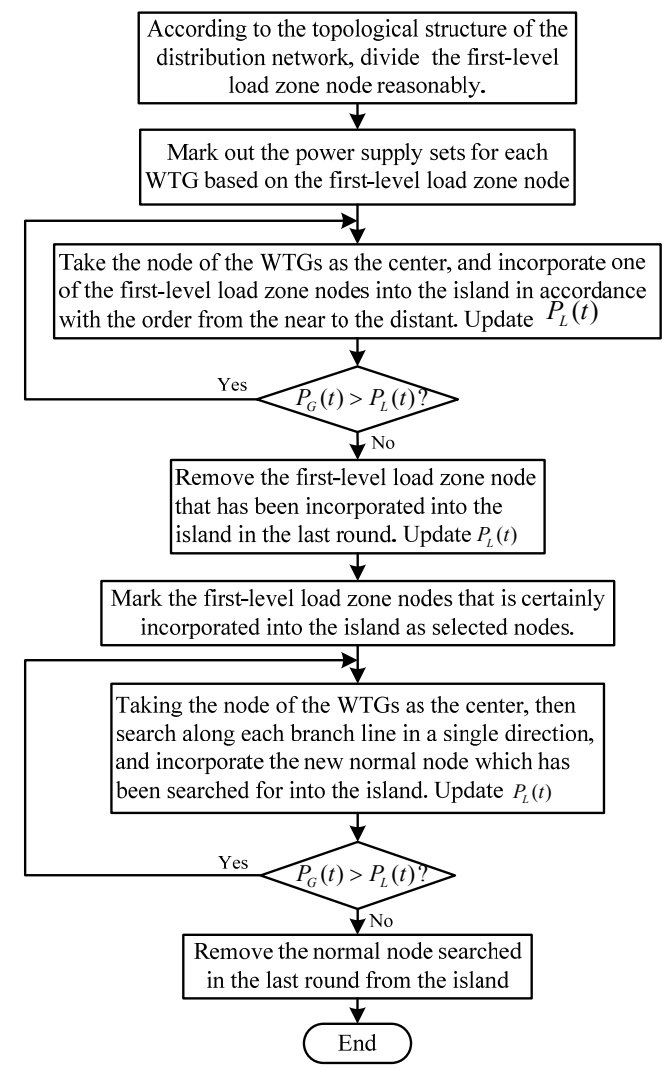

Fig. 5. Flow chart of the dynamic island division procedures is depicted in Fig. 5.

In Fig. 5, $P_{G}(t)$ is the real time output power of WTGs in the island, and $P_{L}(t)$ is the total power that is incorporated into the island.

In addition, if several first-level load zone nodes are attached to one feeder line, the calculation of the supplementary loads should not be repeated. If the achieved island collections have intersecting parts, two island collections can be connected into a larger island collection.

With the dynamic division strategy for island mode, we can simplify the complex power distribution network by adopting the first-level load zone node. During dynamic island division, the proposed method can first mark off each first-level load zone node in the distribution network, then mark out the supply power sets of the first-level load zone node offline for each WTG, and finally, determine the island range online according to the output power of the WTGs. The proposed method can improve the speed of island division, and ensure the power supply of first-level loads. It is particularly suitable for a distribution system with large capacity distributed power.

\section{Failure Mode Analysis of Distribution System}

\subsection{Main concept of the failure analysis}

Define the switch-section as having identical regional attributes (section type, switch number, switch node, feeder branch, feeder node, reliability parameters), where there are mapping relations among the section type, switch number and switch node. Meanwhile, we define a corresponding storage pointer for regional attribute. Hence, we consider the failure mode analysis based on the regional

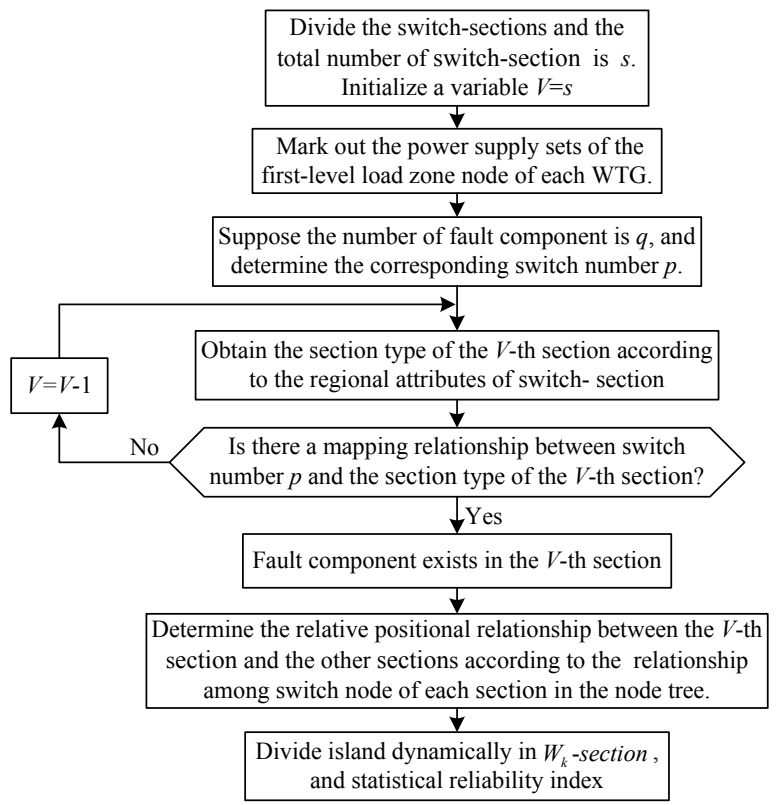

Fig. 6. Flow chart of the failure mode analysis 
attributes of the switch-section.

When a fault occurs in the branch line of a feeder, it is isolated with an automatic switching device in the branch line. It therefore has no effect on the system, and the reliability index can be directly calculated. In this paper, we focus on the failure mode analysis process when a fault occurs in the feeder.

The flow chart of the main concept of the failure mode analysis is depicted in Fig. 6.

\subsection{Examples of the failure mode analysis}

Fig. 7 presents a typical distribution network with WTGs, which contains four circuit-breakers and some isolating switches (the isolating switches are not shown on the child feeders). Node 0 represents the system source node, and nodes 4 and 5 represent WTG access points.

Based on the search method described in section 3, any distribution system can be simplified to a switch-section network. Fig. 8 depicts the switch-section network that corresponds to Fig. 7.

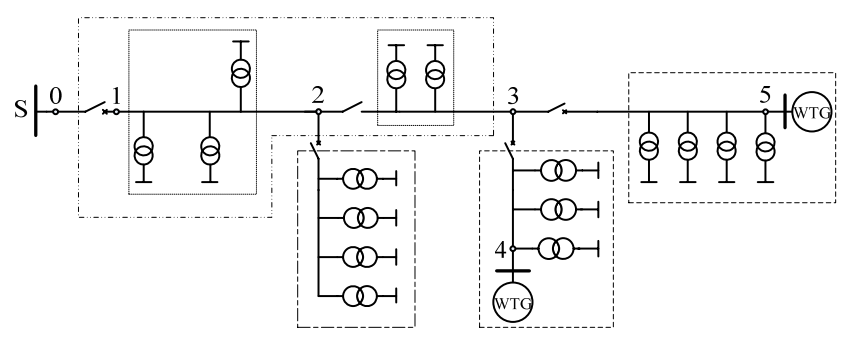

Fig. 7. Typical distribution network with WTGs

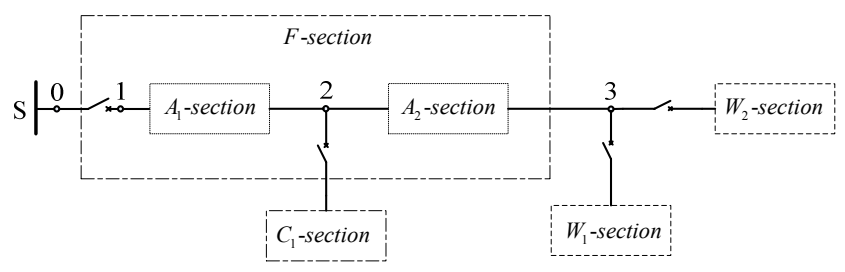

Fig. 8. Switch-section network

When a fault occurs in the $F$-section, identify whether the fault occurs in the $A_{1}$-section or $A_{2}$-section. If the fault occurs in the $A_{1}$-section, the power supply in this section cannot be restored before the failure is repaired because the $A_{2}$-section and $C_{1}$-section are located downstream from the fault section. Thus, the outage time of the load is the fault-repair time. Meanwhile, $W_{1}$-section and $W_{2}$-section immediately transfer to island-operation mode as breakers, and the operation time of the island becomes the fault-repair time. In this period, the power supply is constrained by the total load and output power of WTGs in the island. If the fault occurs in the $A_{2}$-section, the section cannot be restored before the fault is isolated because the $A_{1}$-section and $C_{1}$-section are located upstream from the fault section, so the outage time of all loads becomes the fault-isolation time. The analytical process for the $W_{1}$-section and $W_{2}$-section is identical to the analytical process for the $A_{1}$-section. For the $A_{i}$-section $(i=1,2)$ where a fault occurs, the power supply of the load node must be restored after the failure recovery because of the restrictions imposed by the internal structure.

When a fault occurs in the $C_{1}$-section or $W_{i}$-section $(i=1,2)$, the circuit breaker immediately trips into fault isolation, so the fault has no effect on other sections in the system. However, when a fault occurs in the $C_{1}$-section, the outage time of the load downstream from the failure becomes the fault repair time because the section does not contain the WTGs and the standby power supply, and the outage time of the upstream load becomes the faultisolation time. When the fault occurs in the $W_{i}$-section, it immediately switches into island-operation mode, and the power supply condition of the load becomes constrained by the total amount of the load, output power of WTGs and locations of WTGs.

In some cases (such as when a fault occurs in the $A_{1}$-section ), especially when the repair time is relatively long, if the power capacity of WTGs is large enough, the circuit breaker can be operated to enable the $W_{1}$-section and $W_{2}$-section to supply power together, which can improve the power supply range.

\section{Reliability Evaluation of Distribution Network with WTGs}

We apply the sequential Monte Carlo method to simulate the reliability evaluation. One condition for loop termination in this method requires as an input the accumulation of time to failure of a certain element. As such, the sequential

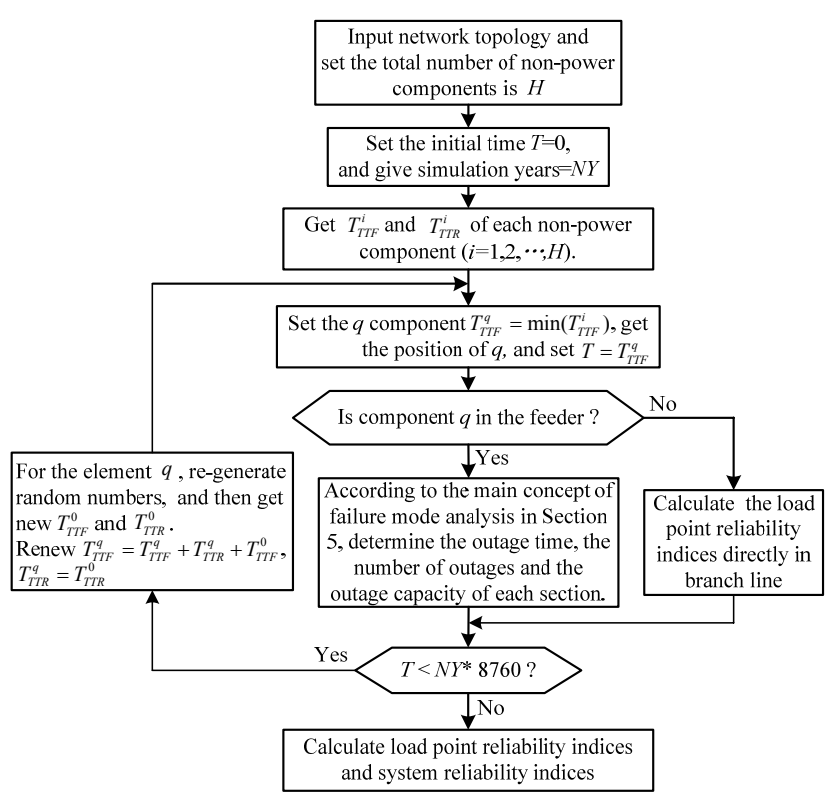

Fig. 9. Reliability evaluation flow chart of distribution system with WTGs 
Monte Carlo method can increase the number of required samplings in the simulation process to achieve a more reliable index and more authentically simulate the fault state of the distribution network in the simulation period.

The reliability evaluation flow chart for a distribution system with WTG is displayed in Fig. 9. In Fig. 9, $T_{T T F}^{i}$ is the time to failure for each non-power element, and $T_{T T R}^{i}$ is the time to repair for each non-power element.

\section{Numerical examples}

\subsection{Example of the system}

As an example, we take the improved IEEE - RBTS Bus6 system [19]. We set the main feeder F4 as the foundation to study the reliability of the distribution network with WTGs. The example system is shown in Fig. 10. As shown in Fig. 10(a), the system contains four sets of circuit breakers, many isolating switches, 23 load points (LP1 to LP23) and 23 distribution transformers. We consider the transformer failure rate to be equivalent to the line and set the LP20 as the first-level load. So there are two first-level loads: LP18 and LP20. All loads adopt a time-varying load model [20], and the circuit-breakers and isolating switches are $100 \%$ reliable. The rated output power of the WTGs is 1.2 MW. The cut-in wind speed, rated wind speed and cut-out wind speed of the WTGs are $3 \mathrm{~m} / \mathrm{s}, 14 \mathrm{~m} / \mathrm{s}$ and $22 \mathrm{~m} / \mathrm{s}$, respectively.

\subsection{Switch-section division of the example system}

When WTG1 is added to node 14, WTG2 and WTG3 are added to nodes 18 and 23 in the example system, respectively. Based on the switch-section search method described earlier, the example system in Fig. 10(a) can be simplified to the distribution section in Fig. 10(b). In Fig. 10 (b), the numbers show the load units nodes, where nodes 20 and 22 are the load units with the first-level load. If the power supplies of the identical first-level load are different, then the supplementary loads of the zone nodes are different as well. Due to economic factors, we do not use WTGs to supply power over a long distance. Therefore, each first-level load zone node can be described as follows: $T_{1-20}=[17,18,19,20], T_{2-22}=[22,23]$, and the power supply nodes of the first-level load can be described as follows: WTG2 is for $T_{1-20}$, and WTG3 is for $T_{2-22}$, where WTG1, WTG2 and WTG3 should guarantee the power supply of the load within their zone. In certain fault conditions, if the unit capacity has a surplus, some sections can join the power supply through switch operation after fault isolation, which can expand the scope of the island

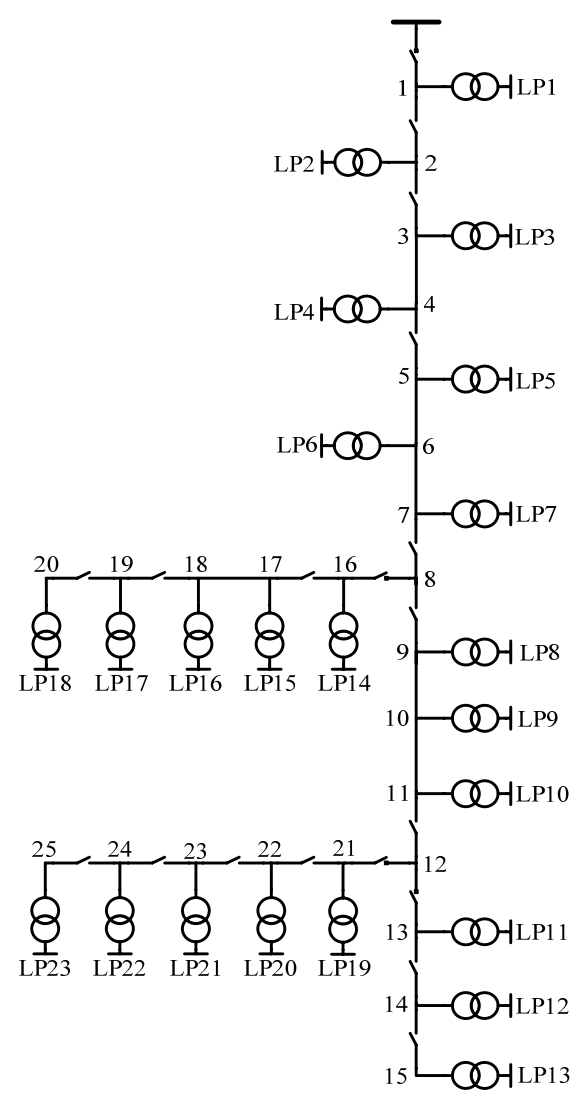

(a) Original example system

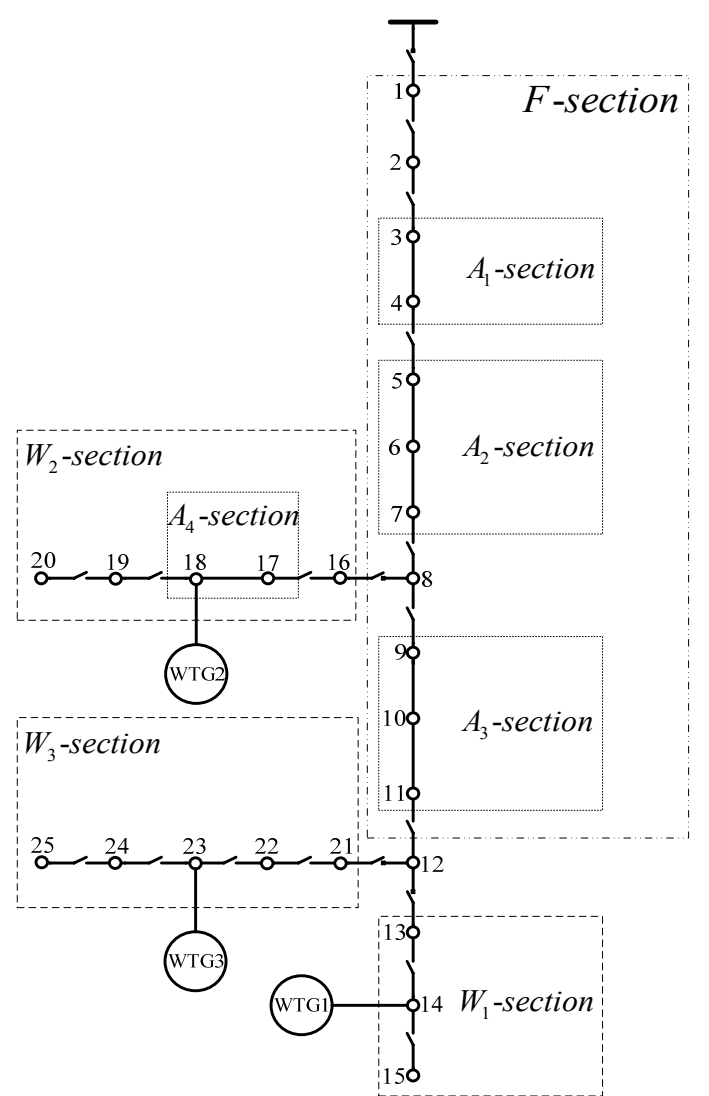

(b) Section division of the system

Fig. 10. Example system 
Table 1. Load point reliability indices

\begin{tabular}{c|c|c|c|c|c|c|c|c|c|c}
\hline Reliability index & WTG location & LP1 & LP6 & LP13 & LP14 & LP16 & LP18 & LP19 & LP20 & LP23 \\
\hline \multirow{3}{*}{$\lambda_{i /(\mathrm{f} / \mathrm{yr})}$} & None & 1.32 & 1.35 & 1.58 & 1.93 & 1.93 & 1.93 & 1.91 & 1.95 & 1.78 \\
\cline { 2 - 12 } & Node 20 & 1.32 & 1.35 & 1.58 & 1.93 & 1.93 & 1.93 & 1.91 & 1.95 & 1.78 \\
\cline { 2 - 13 } & Node 25 & 1.32 & 1.35 & 1.58 & 1.93 & 1.93 & 1.93 & 1.91 & 1.95 & 1.78 \\
\hline \multirow{3}{*}{$r_{i /(\mathrm{h} / \mathrm{f})}$} & None & 2.13 & 3.46 & 5.32 & 3.21 & 3.64 & 4.20 & 4.29 & 4.55 & 5.30 \\
\cline { 2 - 12 } & Node 20 & 2.13 & 3.46 & 5.32 & 3.08 & 3.34 & 3.54 & 4.29 & 4.55 & 5.30 \\
\cline { 2 - 11 } & Node 25 & 2.13 & 3.46 & 5.32 & 3.21 & 3.64 & 4.20 & 4.08 & 4.27 & 4.37 \\
\hline \multirow{3}{*}{$U_{i /(\mathrm{h} / \mathrm{yr})}$} & None & 2.81 & 4.66 & 8.41 & 6.20 & 7.03 & 8.10 & 8.19 & 8.84 & 9.44 \\
\cline { 2 - 11 } & Node 20 & 2.81 & 4.66 & 8.41 & 5.94 & 6.45 & 6.83 & 8.19 & 8.84 & 9.44 \\
\cline { 2 - 11 } & Node 25 & 2.81 & 4.66 & 8.41 & 6.20 & 7.03 & 8.10 & 7.79 & 8.30 & 7.78 \\
\hline
\end{tabular}

improving the system reliability index.

\subsection{Analysis of the load point reliability index}

To verify the effect on the system reliability of the load point that is connected to the access of WTGs, we design three cases: without WTG, WTG on node 20 and WTG on node 25 . Table 1 shows the load point reliability indices.

Table 1 shows that the WTG has no effect on the load point failure rate. Because the load point set $L_{1}$ : [LP14, LP16, LP18] and load point set $L_{2}$ : [LP19, LP20, LP23] belong to different island regions, Table 1 shows that, with the WTGs, the load point failure outage time $r_{i}$ and average annual outage time $U_{i}$ decrease inside the island, which can improve the system reliability index. However, it has no effect on the load point reliability index outside the island. Load point LP19 is at the edge of the island range, so the improvement of the reliability index is not substantial. The reliability index of the first-level load (LP20) significantly improves, but this improvement is less than LP23 near the WTGs, which is caused by the system configuration used in this example.

\subsection{Analysis of the system reliability index}

\subsubsection{Effect of different numbers of WTGs on the reliability index}

To verify the effect of different numbers of WTGs on the system reliability index, different cases are designed on nodes 15, 20, 25 as follows: without WTG, one WTG, two WTGs and three WTG. Fig. 11 shows the curve of the system reliability indices.

In Fig. 11, all system reliability indices show a certain degree of improvement except the SAIFI because SAIFI is independent of the integrated state of WTGs. With an increasing number of WTGs, the scope of the island increases and the loss of power of the load point decreases, which further improves the system reliability. However, when the number of WTGs is more than 10, the system reliability index curve tends to be flat and the degree of system reliability improvement decreases until it reaches a saturated state, so we require a comprehensive variety of factors to determine the number of WTGs in which to invest.

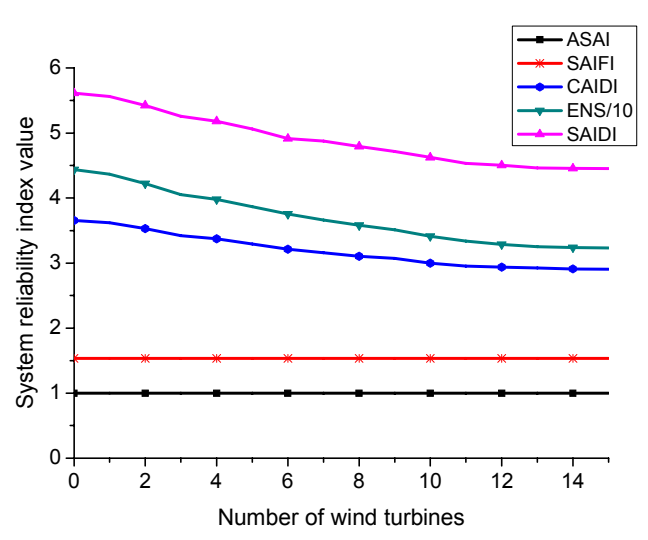

Fig. 11. Curve of system reliability indices for different numbers of WTGs

\subsubsection{Effect of different locations of WTGs on the reliability index}

The installation locations of WTGs also have different effects on the system reliability, so two access cases are described.

Case 1: WTGs are simultaneously installed at nodes 15 , 20, and 25.

Case 2: WTGs are simultaneously installed at nodes 14, 18, and 23.

From Table 2, Case 2 results in an improvement in the system reliability index over Case 1 . The reasons are mainly as follows:

Table 2. Reliability indices with different WTGs locations

\begin{tabular}{c|c|c|c|c|c}
\hline Case & $\begin{array}{c}\text { SAIFI } \\
\text { (f/syst.cust) }\end{array}$ & $\begin{array}{c}\text { SAIDI } \\
(\mathrm{f} / \text { syst.cust })\end{array}$ & $\begin{array}{c}\text { CAIDI } \\
(\mathrm{h} / \text { cust })\end{array}$ & $\begin{array}{c}\text { ASAI } \\
(\%)\end{array}$ & $\begin{array}{c}\text { ENS } \\
(\mathrm{MW} . \mathrm{h} / \mathrm{yr})\end{array}$ \\
\hline Case 1 & 1.536 & 5.256 & 3.422 & 99.9400 & 40.517 \\
\hline Case 2 & 1.536 & 5.187 & 3.376 & 99.9408 & 40.964 \\
\hline
\end{tabular}

1) WTGs are simultaneously installed at nodes 14,18 , and 23 in Case 2, so it is possible to search for the first-level load zone nodes in different directions based on the policy of island division as described previously, and the island range can have various options. The WTGs that are simultaneously installed at nodes 15, 20, and 25 in Case 1 are restricted by the system structure, so the search is only in a single direction: towards the upstream parent node. 
2) Compared to the installation positions in Case 1, when WTGs are installed at nodes 14, 18, and 23, the feeder distance among the three nodes is reduced. As a consequence, an island joint supply can be realized after the fault is isolated under certain fault conditions, which can maximize the use of the output of the WTGs. For example, if the $A_{3}$-section or its upstream section fails, WTG1 and WTG2 can feed in a joint power supply after the fault isolation; if the $A_{2}$-section or its upstream section fails, WTG1, WTG2 and WTG3 can supply power to the $A_{3}$-section together, which improves the system reliability index.

\subsubsection{Effect of different types of power on the reliability index}

To further verify different effects on the system reliability of WTG and non-intermittent power, different cases are designed at nodes 14, 18,and 23 as follows: with three simultaneous WTGs, and with an equal capacity fuel cell. Fig. 12 shows the bar chart of the system reliability indices.

From the bar chart in Fig. 12, it is clear that wind power has far less of an effect than does the large capacity of the fuel cell on the improvement of the system reliability index. This disparity occurs because the wind turbines have randomness and intermittency, which leads to a limited supply capacity, whereas the large capacity fuel cell has no such restrictions. However, because of their good social and environmental benefits, WTGs can play an important role in improving the reliability of the distribution network, particularly after wind power technology has been widely promoted.

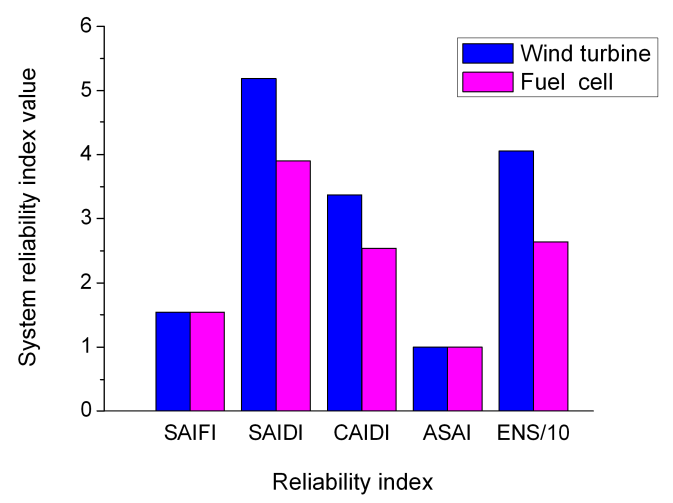

Fig. 12. Bar chart of the system reliability indices for different types of power

\section{Conclusions}

This paper studied the reliability evaluation of a distribution system with WTGs based on the switch-section partitioning method. The main contributions of this paper can be listed as follows:
1) A reliability model of WTGs for the sequential Monte Carlo simulation was established using the analytic method of Weibull distribution and the Markov chain, which is more practical because it includes the derated and stop states.

2) Failure mode analysis for the distribution network with WTGs was studied. The complex distribution network can be divided efficiently based on the switch-section search method. It is possible to reduce the required amount of calculation for the fault effect analysis by applying the regional attributes of the switch-section stored in advance, and the analysis hierarchy can thus become clearer.

3) The sequential Monte Carlo simulation method is adopted to evaluate the reliability of a distribution network with WTGs. By using the accumulation of time to failure of a given element as the condition for loop termination, it is possible to increase the number of samplings required in the simulation process to make the reliability indices more accurate and to simulate the fault state of the distribution network more authentically in the simulation period.

4) The access of WTGs can improve the reliability index of the part load point and system reliability index. However, WTGs are less effective than non-intermittent distributed generation in improving the system reliability. Taking into account the effect of the island division strategy, different numbers and locations of WTGs have different effects on improving the system reliability index.

\section{Acknowledgements}

This work is supported by the National High Technology Research and Development Program (863 Program) of China under Grant 2015AA050104, the National Natural Science Foundation of China under Grant 51177036 and the Fundamental Research Funds for the Central Universities.

\section{References}

[1] R. Billinton and P. Wang, "Reliability network equivalent approach to distribution system reliability evaluation," IEE Proceedings: Generation, Transmission and Distribution, vol. 145, no. 2, pp. 149-153, Apr. 1998

[2] K. G. Xie, J. Q. Zhou, and R. Billinton, "Reliability evaluation algorithm for complex medium voltage electric distribution networks based on the shortest path," IEE Proceedings: Generation, Transmission and Distribution, vol. 150, no. 6, pp. 686-690, Nov. 2003

[3] Y. M. Atwa, E. F. EI-Saadany, and A. C. Guise, "Supply adequacy assessment of distribution system including wind-based DG during different modes of 
operation," IEEE Transactions on Power Systems, vol. 25, no. 1, pp. 78-86, Mar.2010

[4] I.S. Bae and J.O. Kim, "Reliability evaluation of distributed generation based on operation mode," IEEE Transactions on Power Systems, vol. 22, no. 2, pp. 785-790, May.2007

[5] D. Zhu, R.P. Broadwater, and K.S. Tam, "Impact of DG placement on reliability and efficiency with time-varying loads," IEEE Transactions on Power Systems, vol. 21, no. 1, pp. 419-427, Feb.2006

[6] P. Wang and R. Billinton, "Time-sequential simulation technique for rural distribution system reliability cost/worth evaluation including wind generation as alternative supply," IEE Proceedings: Generation, Transmission and Distribution, vol. 148, no. 4, pp. 355-360, Jul.2001

[7] R. Billinton, H. Chen and R. Ghajar, "A sequential simulation for adequacy evaluation of generation systems including wind energy," IEEE Transactions on Energy Conversion, vol. 11, no. 4, pp. 728-734, Dec.1996

[8] G. Desrochers, M. Blanchard and S. Sud, "A MonteCarlo simulation method for the economic assessment of the contribution of wind energy to the power systems," IEEE Transactions on Energy Convers, vol. 1, no. 4, pp. 50-56, Dec.1986

[9] A.S. Dobakhshari and M. Fotuhi-Firuzabad, "A reliability model of large wind farms for power system adequacy studies," IEEE Transactions on Energy Conversion, vol. 24, no. 3, pp. 792-801, Sept. 2009

[10] A.P. Leite and C.L.T. Borges, "Probabilistic wind farms generation model for reliability studies applied to Brazilian sites," IEEE Transactions on Power Systems, vol. 21, no. 4, pp. 1493-1501, Nov. 2006

[11] A.A. Chowdhury. "Reliability models for large wind farms in generation system planning," IEEE Power Engineering Society General Meeting, 2005, pp. 1926-1933

[12] Y. M. Atwa and E. F. El-Saadany, "Reliability evaluation for distribution system with renewable distributed generation during islanded mode of operation," IEEE Transactions on Power Systems, vol. 24, no. 2, pp. 572-581, May.2009

[13] S. Cui, A. Mohan, and D.S. Weile, "Pareto optimal design of absorbers using a parallel elitist nondominated sorting genetic algorithm and the finite element-boundary integral method," IEEE Transactions on Power Systems, vol. 53, no. 6, pp. 2099-2107, Jun.2005

[14] K. Xie, J. Zhou, and R. Billinton, "Fast algorithm for the reliability evaluation of large-scale electrical distribution networks using the section technique," IEE Proceedings: Generation, Transmission and Distribution, vol. 2, no. 5, pp. 701-707, Sep.2008

[15] D. O. Koval, "Zone-branch reliability methodology for analyzing industrial power systems," IEEE Transactions on Industry Applications, vol. 36, no. 5, pp. 1212-1218, Oct. 2000

[16] J.K. Lin and X.D. Wang. "Reliability Evaluation for Distribution System with Distribute Generation," Power and Energy Engineering Conference (APPEEC), Asia-Pacific, 2010.

[17] Y.Y. Hong and K.L. Pen, "Optimal VAR planning considering intermittent wind power using markov model and quantum evolutionary algorithm," IEEE Transactions on Power Delivery, vol. 25, no. 4, pp. 2987-2996, Oct.2010

[18] W. H. Kersting, W. H. Phillips, and R. C. Doyle, "Distribution feeder reliability studies," IEEE Transactions on Industry Applications, vol. 35, no. 2, pp. 319-323, Mar.1999.

[19] R. Billinton and S. Jonnavithula, "A test system for teaching overall power system reliability assessment," IEEE Transactions on Power Systems, vol. 11, no. 4, pp. 1 670-1676, Dec.1996

[20] "IEEE Reliability Test System Task Force. The IEEE reliability test system," IEEE Transactions on Apparatus and Systems, vol. 98, no. 6, pp. 2047-2054, Dec.1979

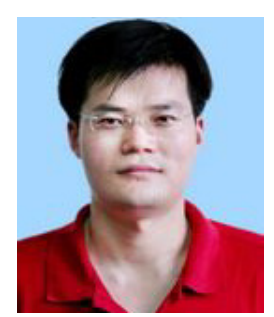

Hongbin Wu He received his B.Sc., M.Sc. and Ph.D. degrees in Electrical Engineering from Hefei University of Technology, Hefei, China in1994, 1998 and 2005 respectively. $\mathrm{He}$ is now a professor of Hefei University of Technology. His research field includes distributed generation technology and distribution network modeling and simulation.

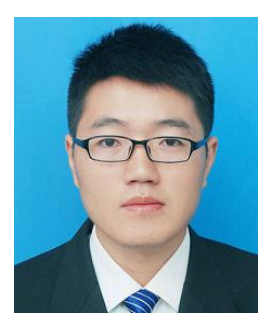

Jinjin Guo He received his B.S. degrees in electrical engineering and automation from Hefei University of Technology, Hefei, China, in 2013. He is now purchasing his M.S. degrees in power system and automation from Hefei University of Technology, Hefei, China. His research interests include distributed power generation technology.

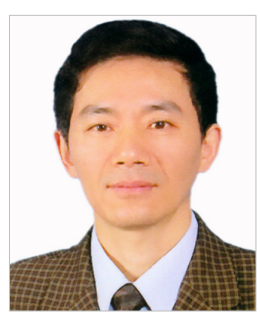

Ming Ding He was born in 1956. He is now a professor of Hefei University of Technology. His research areas include power system reliability, planning, power market and renewable energy resource techniques. 